\title{
Research
}

\section{Etiologies des pleurésies exsudatives: à propos de 424 cas à Madagascar}

\author{
Joëlson Lovaniaina Rakotoson ${ }^{1, \&}$, Radonirina Lazasoa Andrianasolo², Jocelyn Robert Rakotomizao ${ }^{1}$, Hanta Marie Danielle \\ Vololontiana ${ }^{3}$, Kiady Ravahatra ${ }^{1}$, Jobeline Rajaoarifetra ${ }^{1}$, Ange Christophe Félix Andrianarisoa ${ }^{1}$
}

${ }^{1}$ Unité de soins, de formations et de recherches de Pneumologie du Centre Hospitalier Universitaire d'Antananarivo, Madagascar, ${ }^{2}$ Unité de soins, de formations et de recherches de Maladies Infectieuses du Centre Hospitalier Universitaire d'Antananarivo, Madagascar, ${ }^{3} U n i t e ́$ de soins, de formations et de recherches de Médecine Interne du Centre Hospitalier Universitaire d'Antananarivo, Madagascar

${ }^{\&}$ Corresponding author: Joëlson Lovaniaina Rakotoson, Unité de soins, de formations et de recherches de Pneumologie du Centre Hospitalier Universitaire d'Antananarivo, Lot III E 87 Bis AF Mahamasina Sud Antananarivo, Madagascar

Key words: Tuberculose pleurale, biopsie pleurale, tumeurs pleurales, pleurésies d'exsudatives

Received: 22/06/2011 - Accepted: 25/07/2011 - Published: 31/07/2011

\section{Abstract}

Introduction: La pleurésie constitue un motif fréquent de consultation en pneumologie. Notre travail a pour objectif de déterminer les étiologies des pleurésies exsudatives afin d'en faciliter les démarches étiologiques. Méthodes: Il s'agit d'une étude rétrospective réalisée chez des patients ayant une pleurésie exsudative et bénéficiant une biopsie pleurale à l'aveugle à l'aide de l'aiguille de Castelain, pendant une période de 5 ans (2005 à 2009). Résultats: Parmi les 424 patients inclus, 259 hommes (61,08\%) et 165 femmes (38,91\%) étaient individualisés. Les pleurésies étaient d'origine tuberculeuse dans 298 cas (70,28\%), métastatique dans 63 cas (14,85\%), inflammation non spécifique dans 51 cas (12,02\%). Des fibres musculaires striées étaient biopsiées dans 12 cas (2,83\%). Conclusion: La biopsie pleurale occupe une place prépondérante dans la recherche étiologique des pleurésies d'exsudatives à Madagascar où la tuberculose sévit encore en mode endémique.

\section{Pan African Medical Journal. 2011; 9:35}

This article is available online at: http://www.panafrican-med-journal.com/content/article/9/35/full/

(c) Joëlson Lovaniaina Rakotoson et al. The Pan African Medical Journal - ISSN 1937-8688. This is an Open Access article distributed under the terms of the Creative Commons Attribution License (http://creativecommons.org/licenses/by/2.0), which permits unrestricted use, distribution, and reproduction in any medium, provided the original work is properly cited. 


\section{Introduction}

Bien que la thoracoscopie constitue l'examen de choix pour explorer la plèvre, la biopsie pleurale occupe encore une place prépondérante dans les pays en développement. A Madagascar, seule la biopsie pleurale à l'aveugle constitue un moyen d'exploration des pleurésies exsudatives car notre centre hospitalier universitaire (CHU) n'a pas encore à sa disposition une thoracoscopie. C'est pour cela que nous avons essayé d'établir les profils étiologiques des pleurésies exsudatives à partir d'une biopsie pleurale à l'aveugle afin d'améliorer leur prise en charge.

\section{Méthodes}

Nous avons menés une étude rétrospective allant de janvier 2005 à décembre 2009 sur les résultats des examens anatomo-pathologiques d'une biopsie pleurale chez les malades porteurs de pleurésie exsudative. Le caractère exsudatif des pleurésies a été affirmé par la présence du taux de protéine dans le liquide pleural supérieur ou égal à 30 grammes par litre. Tous les malades ayant des pleurésies exsudatives ont bénéficié une biopsie pleurale à l'aveugle à l'aide d'une aiguille de Castelain et les prélèvements obtenus ont été analysé.

\section{Résultats}

Quatre cent vingt-quatre (424) cas de pleurésie exsudative ont été inclus dans notre étude. Nos effectifs étaient composés de 259 hommes $(61,08 \%)$ et 165 femmes $(38,91 \%)$ avec un sex ratio de 1,5 . La moyenne d'âge était de 46,59 ans. La sérologie VIH était négative dans tous les cas. Les étiologies (Tableau 1) étaient constituées par 298 cas de tuberculose (70,28\%), de 63 cas de métastase pleurale (14,85\%) dont 30 cas de métastase d'adénocarcinome, 15 cas de carcinome épidérmoïde, 4 cas de lymphome malin non Hodgkinien, 2 cas de sarcome, 2 cas de carcinome papillaire, métastase de cancer de la thyroïde ( 2 cas), prostate ( 1 cas), rein ( 1 cas), digestif ( 1 cas), bronchique ( 2 cas), sein ( 1 cas), ovaire (1 cas). Cinquante et un cas d'inflammation non spécifique (12,02\%) et 12 cas de fibres musculaires striés (2,83\%) ont été constatés au cours de cette étude.

\section{Discussion}

La tuberculose occupe la première place parmi les étiologies des pleurésies exsudatives à Madagascar, suivie des métastases de cancers avec 9 lésions primitives identifiées. Les résultats de notre étude semblent identiques à ceux des pays à forte prévalence tuberculeuse. La biopsie pleurale à l'aveugle, malgré son faible rendement en cas de pleurésies cancéreuses, tient une place particulière dans l'exploration étiologique des pleurésies exsudatives dans les pays à faible revenu comme Madagascar car notre $\mathrm{CHU}$ n'est pas encore équipé de pleuroscopie. Au Maroc, Berrada et al retrouvaient 251 cas de pleurésies tuberculeuses sur une période de 6 ans dont le diagnostic était confirmé par l'histologie dans $58 \%$ des cas [1]. Dans son étude sur 200 cas de pleurésie, Asriri et al réalisaient la ponction biopsie pleurale dans $84 \%$ des cas et ont constaté une étiologie tuberculeuse dans $52,5 \%$ des cas, bactérienne dans $7 \%$ des cas, néoplasique dans 4,5\% des cas [2]. Bakhatar et al retrouvaient sur 104 cas de pleurésie $36 \%$ de cas de tuberculose, $12 \%$ de métastases, un cas de localisation pleurale d'une leucémie lymphoïde chronique [3] et $8 \%$ d'étiologie bactérienne [3]. Les métastases constituent la grande majorité des tumeurs pleurales [4]. Elles représentent la première cause d'épanchement pleural après 50 ans [4]. Le point de départ est le plus souvent thoracique, et $60 \%$ des métastases pleurales ont pour origine un cancer broncho-pulmonaire ou un cancer du sein [4]. Elles sont surtout représentées par les métastases des adénocarcinomes, des carcinomes malpighiens, des carcinomes à petites cellules, ou encore des mélanomes [5]. Les tumeurs primitives de la plèvre les plus fréquentes sont les tumeurs solitaires fibreuses, les mésothéliomes et les sarcomes, les tumeurs malignes constituant seulement 2 à $3 \%$ de toutes les tumeurs pleurales [5]. Aucune lésion tumorale primitive de la plèvre n'a été identifiée au cours de notre étude. Idéalement, les prélèvements histologiques sont obtenus par biopsie pleurale à l'aveugle, sous pleuroscopie, ou guidée par tomodensitométrie. La biopsie pleurale guidée par tomodensitométrie, à l'aiguille biopsique automatique, a une valeur prédictive positive de $100 \%$, une valeur prédictive négative de $75 \%$ et une précision de $91 \%$ pour le diagnostic de malignité dans la série d'Adams [4]. Mais malgré notre expérience en matière de biopsie pleurale à I'aveugle, qui est également opérateur dépendant, 12 cas de fibres musculaires striés (2,83\%) ont été prélevés.

\section{Conclusion}

La biopsie pleurale à l'aveugle peut être proposée devant le bilan étiologique d'un épanchement pleural exsudatif compatible avec une tuberculose (pays à incidence élevée, épanchement lymphocytaire, intra-dermo-réaction à la tuberculine positive) ou avec une pathologie néoplasique déjà identifiée.

\section{Conflit d'intérêts}

Les auteurs déclarent n’avoir aucun conflit d'intérêts.

\section{Contributions des auteurs}

Tous les auteurs ont participé à la prise en charge des patients et ont lu et approuvé la version finale de l'article. 
Tableau 1: étiologies des pleurésies exsudatives à Madagascar sur un échantillon de 424 cas

\section{Références}

1. Z Berrada, A Aichane, L Sabir, S Safieddine, A Hebbazi, H Afif, Z Bouayad. Pleurésies tuberculeuses. Rev Mal Respir. 2007;24:1S28

2. A Asriri, A Mahmal. Les pleurésies dans un service de pneumologie : à propos de 200 cas. Rev Mal Respir. 2006;HS1(23):89

3. A Bakhatar, F Loudadssi, I Haloui, M El Biaze, H Rachid, F Bourkadi. Les pleurésies au service de pneumologie (à propos de 104 cas). Rev Mal Respir. 2004;HS1(21):80

4. M Brauner, PY Brillet. Autres tumeurs pleurales malignes. Rev Pneumol Clin. 2006;62:124-127. This article on PubMed

5. D Salameire, MH Laverrière, G Ferretti, PY Brichon, S Lantuéjoul. Anatomopathologie des tumeurs et pseudo-tumeurs primitives pleurales. Rev Pneumol Clin. 2006;62:80-86. This article on PubMed

6. T Gentina, A Tremblay, A Brichet, P Ramon, CH Marquette. Les biopsies pleurales à l'aveugle. Rev Mal Respir. 2002;19:533-536. This article on PubMed 
Tableau 1: étiologies des pleurésies exsudatives à Madagascar

\begin{tabular}{lcc}
\hline Etiologies & Nombre & Pourcentage \\
\hline Tuberculose & 298 & 70,28 \\
Métastases & $\mathbf{6 3}$ & $\mathbf{1 4 , 8 5}$ \\
Adénocarcinome & 30 & 47,61 \\
Carcinome épidérmoïde & 15 & 23,80 \\
LMNH & 4 & 6,34 \\
Fibrosarcome & 2 & 3,17 \\
Carcinome papillaire & 2 & 3,17 \\
Cancer thyroïde & 2 & 3,17 \\
Cancer digestif & 2 & 3,17 \\
Cancer bronchique & 2 & 3,17 \\
Cancer prostate & 1 & 1,58 \\
Cancer rein & 1 & 1,58 \\
Cancer sein & 1 & 1,58 \\
Cancer ovaire & 1 & 1,58 \\
Inflammation non spécifique & & $\mathbf{1 2 , 0 2}$ \\
Fibre musculaire strié & $\mathbf{5 1}$ & $\mathbf{2 , 8 3}$ \\
\hline LMNH:LymhomeMaln Non & $\mathbf{1 2}$ & \\
\hline
\end{tabular}

